\title{
Aplicação da transformação raiz quadrada-conformação na análise de estabilidade de escoamentos viscoelásticos
}

\author{
Beatriz L. Carreira ${ }^{1}$ \\ Analice C. Brandi ${ }^{2}$ \\ FCT/Unesp, Presidente Prudente, SP \\ Laison J. S. Furlan ${ }^{3}$ \\ Matheus T. Araujo ${ }^{4}$ \\ Leandro F. Souza ${ }^{5}$ \\ ICMC/USP, São Carlos, SP
}

\begin{abstract}
Resumo. Uma das dificuldades encontradas no tratamento de escoamentos viscoelásticos é o Problema do Alto Número de Weissenberg. Essa limitação consiste no surgimento de instabilidades ou na não-convergência da solução decorrentes de um colapso dos esquemas numéricos. Neste sentido, o objetivo deste trabalho é aplicar a transformação raiz quadrada como técnica estabilizadora do Problema do Alto Número de Weissenberg, a partir da decomposição do tensor conformação na simulação de um escoamento de Poiseuille para um fluido viscoelástico do tipo Giesekus. A Simulação Numérica Direta foi utilizada para investigar a estabilidade hidrodinâmica desse escoamento na transição laminar-turbulenta e comparando com escoamentos Newtonianos.
\end{abstract}

Palavras-chave. Problema do Alto Número de Weissenberg, Tensor Conformação, Simulação Numérica Direta, Fluido Viscoelástico.

\section{Introdução}

O estudo de escoamentos de fluidos viscoelásticos é importante na Reologia Computacional porque muitos materiais usados em aplicações industriais se comportam como fluidos viscoelásticos. Exemplos são produtos automotivos e aeroespaciais, várias embalagens de alimentos, tintas e muitos outros. Esses fluidos são caracterizados por possuírem propriedades viscosas e elásticas ao mesmo tempo, o que torna necessário adicionar uma equação constitutiva para o tensor extratensão não-Newtoniano. Dessa forma, cada vez mais, métodos computacionais são utilizados como ferramenta de modelagem e simulação de escoamentos de interesse no setor industrial.

Este trabalho, em particular, dedica-se à investigação da estabilidade hidrodinâmica do escoamento [2], com o objetivo de prever mudanças que ocorram no regime de escoamento laminar de um fluido levando-o potencialmente ao regime turbulento. Este processo é conhecido como transição laminar-turbulenta, e ainda não é completamente estabelecido, principalmente para escoamentos não-Newtonianos.

Geralmente grandezas adimensionais são empregadas para caracterizar um escoamento. O número de Weissenberg, por exemplo, está relacionado a escoamentos viscoelásticos, e é utilizado

\footnotetext{
${ }^{1}$ beatrizlcarreira@gmail.com

2 analice.brandi@unesp.br

${ }^{3}$ laisonfurlan@usp.br

${ }^{4}$ matheustozo@gmail.com

${ }^{5}$ lefraso@icmc.usp.br
} 
para mensurar o nível de elasticidade do fluido. Porém, um problema de instabilidade numérica surge exatamente a partir deste parâmetro, sendo conhecido na literatura como Problema do Alto Número de Weissenberg ou em inglês "High Weissenberg Number Problem"(HWNP).

Uma das causas do HWNP, segundo a literatura, está relacionada à perda da positividade dos tensores extra-tensão. Tendo em vista que o tensor conformação é simétrico e definido positivo e capaz de manter essas boas propriedades ao longo da evolução temporal do problema, então uma abordagem que surgiu na tentativa de solucionar problema relacionado ao alto número de Weissenberg formula o modelo a partir deste tensor [5].

Recentemente, Afonso et al. [1] apresentaram uma formulação núcleo do tensor conformação para uma grande classe de modelos constitutivos diferenciais, que admite o uso de diversas transformações para decomposição do tensor conformação. Essa técnica, que ficou conhecida como "kernel-conformation" engloba outras formulações específicas e não genéricas que já haviam sido construídas anteriormente.

Sendo assim, neste trabalho aplica-se a transformação raiz quadrada núcleo-conformação para estabilização do HWNP em um escoamento de Poiseuille bidimensional de um fluido viscoelástico Giesekus, utilizando a técnica de Simulação Numérica Direta (DNS). O principal objetivo é investigar a estabilidade hidrodinâmica desses escoamentos, inclusive para altos números de Weissenberg.

\section{Formulação Matemática}

Considera-se um escoamento bidimensional, incompressível, isotérmico e não-Newtoniano, governado pelas equações da continuidade e de conservação do momento, respectivamente,

$$
\begin{gathered}
\nabla \cdot \mathbf{u}=0 \\
\frac{\partial \mathbf{u}}{\partial t}+\nabla \cdot(\mathbf{u u})=-\nabla p+\frac{\beta}{R e} \nabla^{2} \mathbf{u}+\nabla \cdot \mathbf{T},
\end{gathered}
$$

onde $\mathbf{u}$ representa o campo de velocidade, $t$ é o tempo, $p$ é a pressão e $\mathbf{T}$ é o tensor simétrico extra-tensão não-Newtoniano, dado por $\mathbf{T}=\left[\begin{array}{cc}T^{x x} & T^{x y} \\ T^{x y} & T^{y y}\end{array}\right]$.

O parâmetro adimensional $R e=\rho U L / \eta_{0}$ está associado ao número de Reynolds, onde $L$ e $U$ denotam as escalas de comprimento e velocidade, respectivamente, e $\rho$ é a densidade do fluido. A contribuição do solvente Newtoniano é controlada pelo coeficiente adimensional $\beta=\eta_{s} / \eta_{0}$, onde $\eta_{0}=\eta_{s}+\eta_{p}$ representa a viscosidade total do fluido, sendo $\eta_{s}$ e $\eta_{p}$ as viscosidades do solvente Newtoniano e polimérico, respectivamente.

Neste trabalho considera-se o escoamento de fluido viscoelástico governado pela equação constitutiva não linear do modelo Giesekus [4] dada por

$$
\mathbf{T}+W i \stackrel{\nabla}{\mathbf{T}}+\alpha_{G} \frac{W i R e}{1-\beta}(\mathbf{T} \cdot \mathbf{T})=\frac{1-\beta}{R e}\left(\nabla \mathbf{u}+\nabla \mathbf{u}^{\top}\right),
$$

onde $\alpha_{G}$ é o parâmetro de mobilidade que regula o comportamento "shear thinning" do fluido $\left(0 \leq \alpha_{G} \leq 1\right), \mathbf{T} \cdot \mathbf{T}$ denota um produto tensorial e $\stackrel{\nabla}{\mathbf{T}}$ é a derivada convectada. O parâmetro adimensional $W i=\lambda U / L$ é o número de Weissenberg, sendo $\lambda$ o tempo de relaxação do fluido.

\subsection{Formulação Raiz Quadrada-Conformação}

Uma alternativa para descrever os modelos viscoelásticos usa o tensor conformação A. Esse tensor é simétrico e definido positivo e sua equação constitutiva pode ser escrita como

$$
\frac{\partial \mathbf{A}}{\partial t}+\nabla \cdot(\mathbf{u A})=\nabla \mathbf{u A}+\mathbf{A} \nabla \mathbf{u}^{\top}+\frac{1}{W i} f(\mathbf{A}) P(\mathbf{A})
$$


sendo que a relação entre o tensor extra-tensão não-Newtoniano $\mathbf{T}$ e $\mathbf{A}$ pode ser dada por $\mathbf{T}=\xi(\mathbf{A}-\mathbf{I})$, onde $\xi$ é um escalar definido como $\xi=(1-\beta) / R e W i$. A função escalar $f(\mathbf{A})$ e o tensor $P(\mathbf{A})$, são definidos de acordo com o modelo do fluido. Em particular, para o modelo Giesekus $f(\mathbf{A})=1$ e $P(\mathbf{A})=(\mathbf{I}-\mathbf{A})\left[\mathbf{I}+\alpha_{G}(\mathbf{A}-\mathbf{I})\right]$.

Através da decomposição espectral do tensor conformação $\boldsymbol{\Lambda}=\boldsymbol{O}^{\top} \boldsymbol{A} \boldsymbol{O}$, pode-se construir uma equação de evolução para os autovalores da matriz $\boldsymbol{A}$ a partir da equação (4). Posteriormente constrói-se uma equação de evolução para a função núcleo $\mathbb{K}$ avaliada na matriz dos autovalores de $\boldsymbol{A}$, e finalmente, após as devidas manipulações algébricas, a equação de evolução da transformação núcleo aplicada ao tensor conformação é expressa por

$$
\frac{D \mathbb{K}(\boldsymbol{A})}{D t}=\frac{\partial \mathbb{K}(\boldsymbol{A})}{\partial t}+\nabla \cdot(\mathbf{u} \mathbb{K}(\boldsymbol{A}))=(\boldsymbol{\Omega} \mathbb{K}(\boldsymbol{A})-\mathbb{K}(\boldsymbol{A}) \boldsymbol{\Omega})+2 \mathbb{B}+\frac{1}{W i} \mathbb{H},
$$

sendo que os tensores $\mathbb{B}$ e $\boldsymbol{\Omega}$ surgem de uma reformulação do gradiente de velocidade, realizada por $[3]$ e $\mathbb{H}=\boldsymbol{O} P(\boldsymbol{\Lambda}) \boldsymbol{J} \boldsymbol{O}^{\top}$, onde $\boldsymbol{J}$ é a matriz jacobiana de $\mathbb{K}(\boldsymbol{\Lambda})$.

Definindo $\mathbb{Q}=\mathbf{A}^{\frac{1}{2}}$ como a raiz quadrada do tensor conformação, então $\mathbb{Q}^{2}=\mathbf{A}$. Após substituir a função núcleo $\mathbb{Q}$ em (5), uma equação de evolução para a transformação raiz quadrada aplicada ao tensor conformação é finalmente obtida

$$
\frac{\partial \mathbb{Q}}{\partial t}+\nabla(\mathbf{u} \mathbb{Q})=(\boldsymbol{\Omega} \mathbb{Q}-\mathbb{Q} \boldsymbol{\Omega})+\boldsymbol{B} \mathbb{Q}+\frac{1}{2 W i} f\left(\mathbb{Q}^{2}\right) \mathbb{Q}^{-1} P\left(\mathbb{Q}^{2}\right)
$$

\section{Simulação Numérica Direta}

A fim de simplificar o problema e eliminar o tratamento da pressão na equação do momento, utiliza-se a formulação vorticidade-velocidade [2]. A vorticidade $\omega_{z}$, em coordenadas bidimensionais, é matematicamente definida como

$$
\omega_{z}=\frac{\partial u}{\partial y}-\frac{\partial v}{\partial x}
$$

Aplicando tal formulação, o problema agora consiste em resolver o sistema composto pela equação da continuidade (8), equação da vorticidade (9) e transporte de vorticidade (10), além das equações dos tensores não-Newtonianos (11) - (13)

$$
\begin{gathered}
\frac{\partial u}{\partial x}+\frac{\partial v}{\partial y}=0 \\
\frac{\partial^{2} v}{\partial x^{2}}+\frac{\partial^{2} v}{\partial y^{2}}=\frac{\partial \omega_{z}}{\partial x}, \\
\frac{\partial \omega_{z}}{\partial t}+u \frac{\partial \omega_{z}}{\partial x}+v \frac{\partial \omega_{z}}{\partial y}=\frac{\beta}{R e}\left[\frac{\partial^{2} \omega_{z}}{\partial x^{2}}+\frac{\partial^{2} \omega_{z}}{\partial y^{2}}\right]-\frac{\partial^{2} T^{x y}}{\partial x^{2}}-\frac{\partial^{2} T^{y y}}{\partial x \partial y}+\frac{\partial^{2} T^{x x}}{\partial y \partial x}+\frac{\partial^{2} T^{x y}}{\partial y^{2}}, \\
T^{x x}+W i\left(\frac{\partial T^{x x}}{\partial t}+u \frac{\partial T^{x x}}{\partial x}+v \frac{\partial T^{x x}}{\partial y}-2 T^{x x} \frac{\partial u}{\partial x}-2 T^{x y} \frac{\partial u}{\partial y}\right)+\alpha G \frac{W i R e}{1-\beta}\left(T^{x x^{2}}+T^{x y^{2}}\right)=2 \frac{1-\beta}{\operatorname{Re}} \frac{\partial u}{\partial x},(11) \\
T^{y y}+W i\left(\frac{\partial T^{x y}}{\partial t}+u \frac{\partial T^{x y}}{\partial x}+v \frac{\partial T^{x y}}{\partial y}-T^{x x} \frac{\partial v}{\partial x}-T^{y y} \frac{\partial u}{\partial y}\right)+\alpha G \frac{W^{x}}{1-\beta}\left(T^{x y}\left(T^{x x}+T^{y y}\right)\right)=\frac{1-\beta}{\operatorname{Re}}\left(\frac{\partial v}{\partial x}+\frac{\partial u}{\partial y}\right), \\
(12) \\
\left.\frac{\partial T^{y y}}{\partial x}+v \frac{\partial T^{y y}}{\partial y}-2 T^{x y} \frac{\partial v}{\partial x}-2 T^{y y} \frac{\partial v}{\partial y}\right)+\alpha G \frac{W i R e}{1-\beta}\left(T^{x y^{2}}+T^{y y^{2}}\right)=2 \frac{1-\beta}{\operatorname{Re}} \frac{\partial v}{\partial y} .(13)
\end{gathered}
$$




\subsection{Escoamento Base}

Considerando o escoamento de Poiseuille viscoelástico e bidimensional, $x$ representa a direção do escoamento, enquanto y representa a direção normal à parede. Para calcular o escoamento base, assume-se que todas as variáveis são dependentes apenas do eixo $y$, exceto para a pressão cujo gradiente é constante na direção $x$. O domínio na direção $y$ está compreendido entre $[-1,1]$.

No entanto, o sistema de equações que deriva dessas hipóteses não possui uma solução analítica completa disponível na literatura. Sendo assim, o escoamento base foi gerado numericamente por um código DNS bidimensional, sem perturbações, implementado com a formulação raiz quadradaconformação para estabilização do HWNP. As simulações são realizadas até o escoamento atingir o estado estacionário.

\section{Método Numérico}

Na Figura 1 observa que o fluido entra no domínio computacional em $x=x_{0}$ e sai em $x=x_{\max }$. Neste trabalho investiga-se o comportamento das ondas de Tollmien-Schlichting no escoamento, assim, perturbações temporais são introduzidas através da técnica de injeção e sucção de massa na parede. Isso ocorre na região compreendida entre $x_{1}$ e $x_{2}$, após um intervalo de tempo, mais especificamente no tempo $t+\delta t$, impondo a velocidade $v$ como sendo

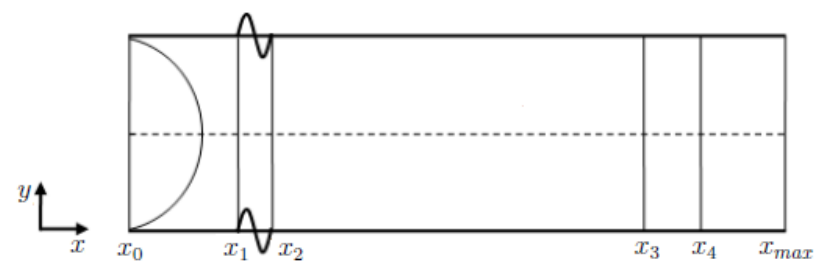

Figura 1: Definição do domínio computacional para o escoamento de Poiseuille.

$$
v=A f(x) \sin \left(\omega_{t} t\right), \quad x_{1}<x<x_{2},
$$

$\mathrm{e}$

$$
v=0, \quad x \leq x_{1} \quad \text { ou } \quad x \geq x_{2},
$$

onde $A$ é o parâmetro que ajusta a amplitude das perturbações, $f(x)$ é uma função polinomial de grau 9 e $\omega_{t}$ é a frequência da perturbação. Técnicas de amortecimento são implementadas a fim de evitar reflexões de ondas nas regiões de entrada (entre $x_{0}$ e $x_{1}$ ) e saída (entre $x_{3}$ e $x_{4}$ ).

A discretização temporal das equações modelo do escoamento, bem como dos tensores nãoNewtonianos é realizada através de um esquema de Runge-Kutta clássico de quarta ordem. Discretizações espaciais para o cálculo das derivadas são realizadas usando métodos de diferenças finitas compactas de alta ordem, mais especificamente, quinta e sexta ordem de precisão [7]. A utilização desses métodos demanda a solução de sistemas lineares tridiagonais. O sistema linear resultante da solução numérica da equação de Poisson é resolvido utilizando um esquema de Aproximação Multigrid (FAS) num ciclo $\mathrm{V}$ composto por 4 níveis [6]. A aplicação de discretizações centradas de alta ordem de precisão também faz necessário a utilização de um filtro espacial a fim de eliminar perturbações espúrias da solução numérica. 


\section{Verificação do Código}

O teste de verificação ocorre para um código DNS que simula o problema de Poiseuille bidimensional, incompressível e isotérmico, considerando o fluido Giesekus, e a transformação raiz quadrada-conformação como técnica estabilizadora para simulações com alto número de Weissenberg. Para tanto, compara-se este código considerando $\alpha_{G}=0$ na equação (3) do modelo Giesekus, com a solução analítica do escoamento nas mesmas condições considerando o modelo Oldroyd-B.

Nas simulações numéricas considera-se o número de pontos no sentido do escoamento e no sentido normal à parede, sendo $i_{\max }=9049$ e $j_{\max }=249$, respectivamente, a distância entre dois pontos consecutivos nas direções $x$ e $y, d x=2 \pi / 16 \alpha_{r}$ e $d y=2 /\left(j_{\max }-1\right)$, respectivamente, sendo $\alpha_{r}$ a parte real do número de onda. Os passos no tempo por período de onda são 400.

A Figura 2 demonstra o comportamento dos tensores não-Newtonianos $T^{x x}$ e $T^{x y}$. Nota-se que os resultados numéricos obtidos com a formulação raiz quadrada-conformação (sqrt-conformação) concordam com a solução analítica do escoamento nas mesmas condições. Tem-se, portanto, um indicativo de que o código implementado é adequado para simular e analisar a estabilidade de escoamentos viscoelásticos do tipo Giesekus.

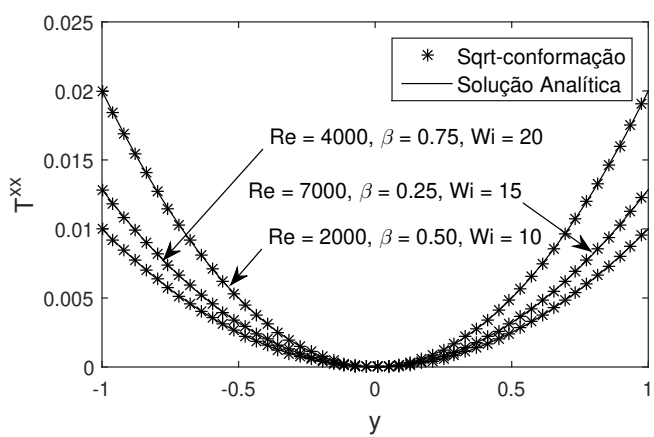

(a)

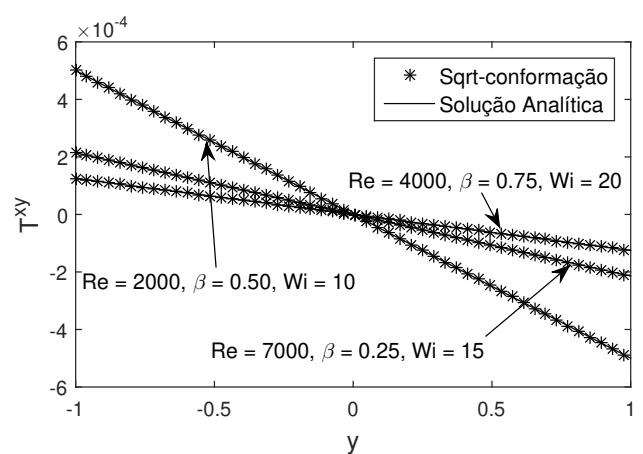

(b)

Figura 2: Solução numérica (Giesekus com $\alpha_{G}=0$ ) e analítica (Oldroyd-B) para o escoamento base: (a) Tensor $T^{x x}$ e (b) Tensor $T^{x y}$.

\section{Resultados Numéricos}

Simulações numéricas são apresentadas a fim de investigar o efeito da constante $\beta$, que controla a contribuição Newtoniana do fluido, na estabilidade hidrodinâmica em um escoamento de Poiseuille do fluido viscoelástico. Considera-se nas simulações: o número de pontos no sentido do escoamento e no sentido normal à parede, $i_{\max }=505$ e $j_{\max }=249$, respectivamente; a distância entre dois pontos consecutivos nas direções $x$ e $y$ são $d x=2 \pi / 16 \alpha_{r}$ e $d y=2 /\left(j_{\max }-1\right)$, respectivamente. Aplicou-se 400 passos no tempo por período de onda, e a frequência de perturbação é $\omega_{t}=0.2$. Para ajuste da amplitude das ondas de Tollmien-Schlichting considerou-se $A=1 \times 10^{-4}$.

Os resultados sobre a influência da constante $\beta$ são apresentados na Figura 3, que contém as curvas que descrevem o comportamento das ondas de Tollmien-Schlichting do fluido Newtoniano e do fluido viscoelástico com seus parâmetros fixados. O comportamento crescente das curvas indica amplificação das perturbações e escoamentos instáveis. Em contrapartida, curvas decrescentes apontam para escoamentos estáveis, com decaimento das perturbações introduzidas.

Na Figura 3 são considerados os seguintes valores para a constante, $\beta=0.50,0.75$ e 0.90 , sendo $R e=5000, \alpha_{G}=0.15$ e variações de Weissenberg $(W i)$. É possível notar principalmente dois efeitos sobre o comportamento dos escoamentos: os acréscimos no número de Weissenberg tornam 


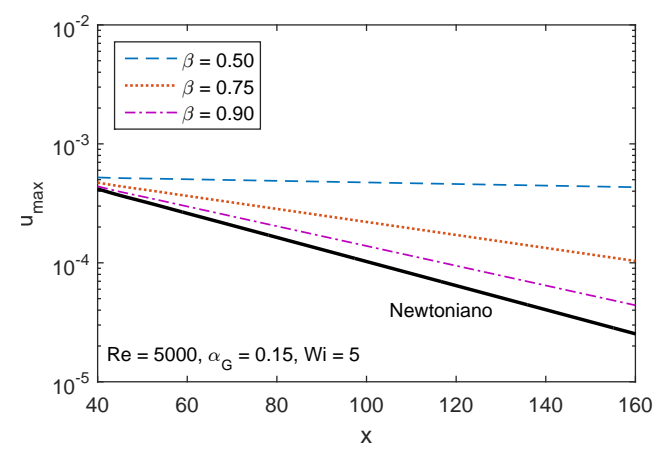

(a)

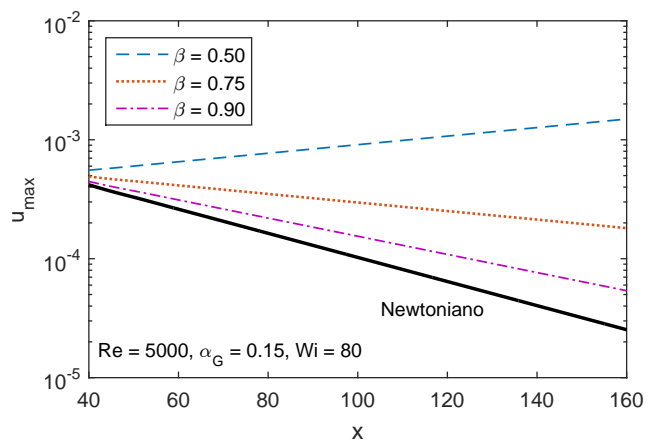

(c)

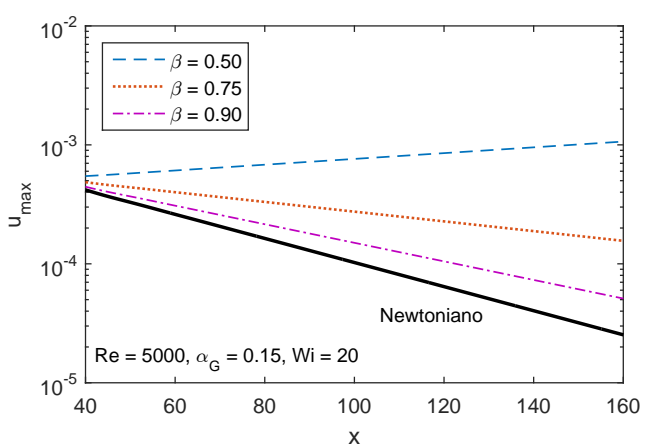

(b)

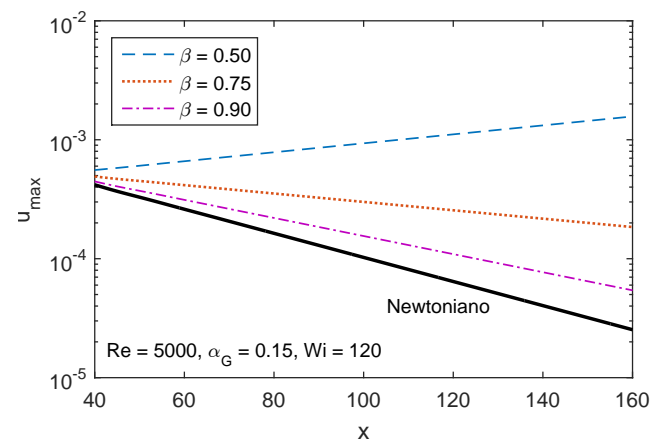

(d)

Figura 3: Valor máximo da perturbação da velocidade na direção do escoamento para diferentes valores de $\beta$ e: (a) $W i=5$; (b) $W i=20$; (c) $W i=80$; e (d) $W i=120$.

os escoamentos menos estáveis considerando $\beta=0.75$ e 0.90 , sendo mais facilmente percebido numa comparação entre as Figuras $3(\mathrm{a})$ e $3(\mathrm{~b})$. Já para $\beta=0.50$, o escoamento que é neutro (Figura 3(a)) torna-se instável com o aumento do número de $W i$.

Percebe-se ainda que as curvas dos escoamentos simulados com o modelo Giesekus vão se aproximando da curva do modelo Newtoniano, conforme o valor de $\beta$ é aumentado. Além disso, em todos os casos, os escoamentos Newtonianos comportaram-se de forma mais estável quando comparados com os escoamentos viscoelásticos, mesmo para os menores valores de $W i$.

A positividade do tensor conformação é uma característica que deve ser preservada durante toda

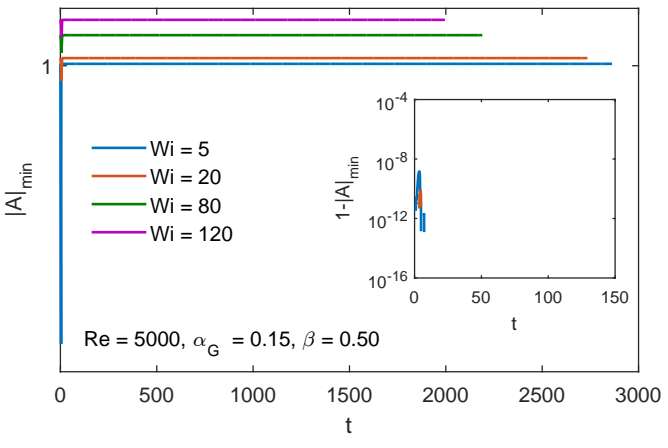

(a)

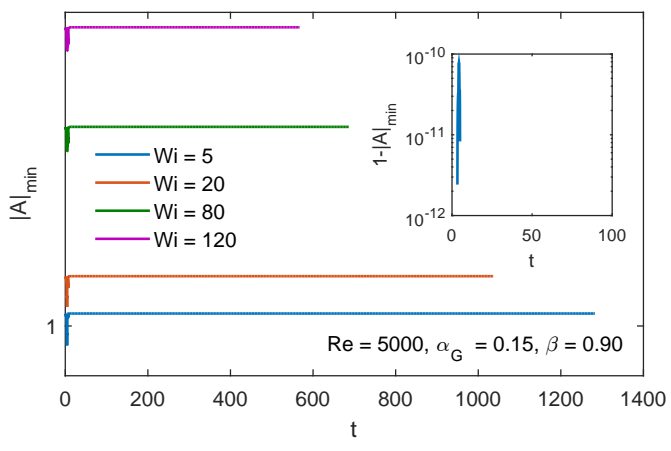

(b)

Figura 4: Evolução temporal do determinante mínimo do tensor conformação. 
evolução temporal da equação constitutiva, necessária para se evitar as instabilidades do HWNP. Segundo Hulsen [5], isso ocorre se o determinante do tensor conformação, satisfizer $|\boldsymbol{A}| \geq 1$.

Neste sentido, a evolução temporal do determinante mínimo do tensor conformação para alguns dos casos simulados é apresentada na Figura 4. Para os menores valores de $W i$ testados há uma pequena oscilação no valor do determinante no início da evolução temporal. Porém, a amplitude dessas oscilações é muito pequena, o que pode ser confirmado através do valor $1-|\boldsymbol{A}|_{\text {min }}$, demonstrando que a transformação sqrt-conformação garante o valor de $|\boldsymbol{A}|_{\text {min }}>1$. Com isso, confirma-se os resultados numéricos anteriormente apresentados para análise de estabilidade do escoamento utilizando esta técnica como estratégia para estabilização do HWNP.

\section{Conclusões}

Neste trabalho, a transformação raiz quadrada-conformação para estabilização do HWNP é apresentada. Considerando o fluido viscoelástico Giesekus, a análise de estabilidade para o escoamento de Poiseuille bidimensional foi realizada utilizando a Simulação Numérica Direta. Para avaliar as taxas de amplificação máximas, diferentes valores dos parâmetros adimensionais foram testados, com atenção especial para a constante $\beta$, responsável por controlar a contribuição Newtoniana no fluido, e também para o número de Weissenberg. Além disso, analisou-se o determinante mínimo do tensor conformação, verificando que os valores obtidos são satisfatórios de acordo com a literatura, garantindo que os resultados referentes à análise de estabilidade são consistentes.

\section{Agradecimentos}

Os autores agradecem à CAPES pelo auxílio financeiro no desenvolvimento deste trabalho.

\section{Referências}

[1] Afonso, A. M., Pinho, F. T. and Alves, M. A. The kernel-conformation constitutive laws, Journal Non-Newtonian Fluid Mechanics, 167-168:30-37, 2012. DOI: 10.1016/j.jnnfm.2011.09.008.

[2] Brandi, A. C., Mendonça, M. T. and Souza, L. F. DNS and LST stability analysis of OldroydB fluid in a flow between two parallel plates, Journal Non-Newtonian Fluid Mechanics, 267:14-27, 2019. DOI: 10.1016/j.jnnfm.2019.03.003.

[3] Fattal, R. and Kupferman, R. Time-dependent simulation of viscoelastic flows at high Weissenberg number using the log-conformation representation, Journal of Non-Newtonian Fluid Mechanics, 126:23-37, 2005. DOI: 10.1016/j.jnnfm.2004.12.003.

[4] Giesekus, H. A simple constitutive equation for polymer fluids based on the concept of deformation-dependent tensorial mobility, Journal of Non-Newtonian Fluid Mechanics, 2:69-109, 1982. DOI: 10.1016/0377-0257(82)85016-7.

[5] Hulsen, M.A. Some properties and analytical expressions for plane flow of Leonov and Giesekus models, Journal Non-Newtonian Fluid Mechanics, 30:85-92, 1988. DOI: 10.1016/03770257(88)80019-3.

[6] Souza, L. F. Instabilidade centrífuga e transição para turbulência em escoamentos laminares sobre superfícies côncavas. Tese de Doutorado, Instituto Tecnológico de Aeronáutica, 2003.

[7] Souza, L. F., Mendonça M. T. and Medeiros M. A. F. The advantages of using high-order finite differences schemes in laminar-turbulent transition studies, International Journal for Numerical Methods Fluids, 48:565-592, 2005. DOI: 10.1002/fld.955. 